\title{
Absorption Enhancement in Si Solar Cells by Incorporation of Metallic Nanoparticles: Improved COMSOL Numerical Study Including Quantum Corrections
}

\author{
K. KluczyK*, Z. KRZemińska and W.A. JaCAK
}

Department of Quantum Technologies, Faculty of Fundamental Problems of Technology, Wrocław University of Science and Technology, Wyb. S. Wyspiańskiego 27, 50-370 Wrocław, Poland

\begin{abstract}
One of the crucial parameters affecting the solar cell efficiency is the absorption rate versus solar spectrum. Metallic nanoparticles deposited on the cell surface can mediate this process. Main mechanisms of absorption enhancement due to metallic nanoparticle plasmons were proposed: (1) the scattering of incident solar light causing increase of the optical path length inside active layer and local enhancement of the electric field; (2) near field coupling between plasmon and semiconductor and the direct generation of electron-hole pairs in the semiconductor. The field concentration effect can be described by classical electrodynamic theory, the coupling between metallic nanoparticle plasmons and band electrons in semiconductor substrate must be captured upon quantum mechanics. In this paper we took the challenge to develop fast and reliable method for calculation of device optical properties by application of COMSOL system appropriately configured to take into account these quantum effects, via the quantum modification of the dielectric function of semiconductor substrate and metallic components. The presented results indicate that the efficiency of energy transfer due to near field coupling of metallic nanoparticle plasmons with semiconductor substrate is much more effective than the absorption increase due to metallic nanoparticle plasmons scattering only.
\end{abstract}

DOI: 10.12693/APhysPolA.132.393

PACS/topics: solar cells, solar energy harvesting, surface plasmons, metallic nanoparticles

\section{Introduction}

The deposition of the metallic nanocomponents on the surface of the solar cell has been proven to increase the overall cell efficiency [1-3]. The proposed mechanisms of these phenomena are connected with [4]: (1) increase of the light path inside the active layer due to scattering on the metallic nanoparticles, (2) near field coupling between plasmons in the metallic nanoparticles and semiconductor band electrons, (3) the direct hot-electron injection to the semiconductor. The first of these mechanisms has been widely studied within classical electrodynamics numerical methods such as finite difference time domain and finite element method [5-7] for various shapes, sizes, and concentrations of arrayed metallic nanocomponents. These calculations were performed using as a prerequisite the material optical parameters taken from various experimental data. The latter are, however, addressed mostly to bulk materials or rarely for thin films and do not display important behavior genuine for nanoparticle size and geometry.

In the present report we compare the absorption enhancement in Si solar cell calculated for material properties taken from the experiment [8] with the one calculated for material properties modeled microscopically within the Fermi golden rule scheme [9]. The numeri-

\footnotetext{
* corresponding author; e-mail katarzyna.kluczyk@pwr.edu.pl
}

cal calculations were carried out by use of the commercial software COMSOL Multiphysics 5.0 implementing finite element method for solving Maxwell equations (http://www.comsol.com). Results are discussed with comparison to experimental data and analytical model for plasmons based on random phase approximation (RPA).

\section{RPA modeling of plasmons in metallic nanoparticles}

Upon the external electromagnetic field the electrons inside the metallic nanoparticles start to oscillate. The single quanta of those oscillations, known as plasmon, can be described within the random phase approximation developed by Pines [10] for bulk metals and accommodated to the case of nanoparticles in [11].

In the proximity of the semiconductor substrate the plasmon oscillations are strongly damped due to the energy transfer between plasmons and band electrons in the semiconductor. The efficiency of this energy transfer can be assessed applying the Fermi golden rule to calculate the photon absorption probability in semiconductor substrate induced by near-field dipole-like coupling between metallic nanoparticle plasmons (MNPs) and semiconductor band electrons. The resulting probability of interband transitions in semiconductor per time unit mediated by MNPs is (see [9]):

$$
\delta w=\frac{4}{3} \frac{\mu^{3 / 2} \sqrt{2} \sqrt{\hbar \omega-E_{g}} e^{2} D_{0}^{2}}{a \hbar^{4} \varepsilon^{2}},
$$

where $a$ is the metallic nanoparticle radius, $D_{0}$ is the 
amplitude of corresponding plasmon dipole, $E_{g}$ is the semiconductor band gap, $\mu=\frac{m_{p} m_{n}}{m_{p}+m_{n}}$ and $m_{n}$ and $m_{p}$ are the effective masses of the electrons and holes, respectively. Notice the difference of above formula from the photon absorption probability calculated for an ordinary photo-effect

$$
\delta w_{0}=\frac{4 \sqrt{2}}{3} \frac{\mu^{5 / 2} e^{2}}{m_{p}^{* 2} \omega \varepsilon \hbar^{3}}\left(\hbar \omega-E_{g}\right)^{3 / 2}\left(\frac{\varepsilon E_{0}^{2} V}{8 \pi \hbar \omega}\right)^{-1} .
$$

The ratio of the probability of photon absorption with and without metallic nanoparticles deposited on the surface of semiconductor defines the photocurrent gain:

$$
\frac{I^{\prime}}{I}=\frac{\beta N_{m}\left(\delta \omega_{0}+\delta \omega\right)}{\delta \omega_{0}}=1+\frac{\beta N_{m} \delta w}{\delta w_{0}},
$$

where $N_{m}$ is the number of metallic nanoparticles and $\beta$ is the factor responsible for all effects that are not directly taken into account (such as deposition separation and surface property perturbations reducing the coupling strength).

In the balanced state we consider the stationary solution of a driven and damped oscillator for MNP modeling, which gives

$$
\frac{\beta N_{m} \delta w}{\delta w_{0}}=\frac{8 \pi a^{2} \beta C_{0} m_{p}^{2} e^{4} n_{e}^{2} \omega^{2} f^{2}(\omega)}{3 \mu m^{2}\left(\hbar \omega-E_{g}\right) \hbar^{2} \varepsilon^{2}},
$$

where $C_{0}=n_{s} 4 \pi a^{3} /(3 H), n_{s}$ is the nanoparticle planar density, $H$ is the depth of Si layer, $f(\omega)=$ $\frac{1}{\sqrt{\left(\omega_{1}^{2}-\omega^{2}\right)^{2}+4 \omega^{2} / \tau^{2}}}, \omega_{1}$ is the resonance frequency of surface plasmons in metallic nanoparticle with radius $a$ and the damping rate $\frac{1}{\tau}$ is calculated by comparison of average power of the oscillator with total power transfer to the semiconductor via photon absorption [9].

\section{Numerical model}

The total dissipated energy, averaged over an optical cycle of an electromagnetic field oscillating at frequency $\omega$ inside a dispersive nonmagnetic material can be calculated according to [12]:

$$
Q=\frac{\omega}{4 \pi}\left(\varepsilon^{\prime \prime}(\omega) \boldsymbol{E}^{2}+\mu^{\prime \prime} \boldsymbol{H}^{2}\right)
$$

In the above formula, one can notice that the important parameter deciding on dissipated energy is the imaginary part of the dielectric function $\varepsilon^{\prime \prime}(\omega)$ of the medium. The dielectric function arises from the definition of the displacement electric field $\boldsymbol{D}=\varepsilon(\omega, \boldsymbol{r}) \boldsymbol{E}$ and incorporates all information on the processes involved in light matter interaction. The phenomenological character of this function and its connection with the microscopic phenomena allows to model of non-classical quantum effects using the fully classical electromagnetic calculations taking advantage from the Maxwell equations solution in a numerical way for complex system or even whole device without details of their microscopy compressed into the dielectric functions of the system components.

The imaginary part of the dielectric function of the Si substrate equals to

$$
\varepsilon^{\prime \prime}=\frac{n c}{\omega} \alpha(\omega),
$$

where $n$ is the real part of the refractive index and $\alpha$ is the absorption coefficient of the semiconductor substrate. It can be calculated as the ratio of the power absorbed in the substrate to the total power incident on the device calculated according to Eqs. (1) and (2). The amplitude of plasmon oscillations $D_{0}$ in Eq. (1) can be obtained from the formula for total power of the radiation

$$
D_{0}^{2}=\frac{4 \pi \varepsilon_{0} \lambda^{4}}{(2 \pi)^{4} c} \int_{\Sigma} \boldsymbol{S} \cdot \mathrm{d} \boldsymbol{\sigma},
$$

where $\boldsymbol{S}$ is the Poynting vector and $\Sigma$ is the surface of the metallic nanoparticle. The absorption enhancement $\mathcal{A}$ may be defined as the ratio of absorption with and without metallic nanoparticles and can be calculated according to Eq. (5) by integrating the square of the electric field component over the semiconductor substrate volume

$$
\mathcal{A}(\omega)=\frac{\frac{\omega}{4 \pi} \int_{V} n \varepsilon_{m}^{\prime \prime}(\omega) \boldsymbol{E}_{\text {with NP }}^{2} \mathrm{~d} V}{\frac{\omega}{4 \pi} \int_{V} n \varepsilon_{0}^{\prime \prime}(\omega) \boldsymbol{E}_{\text {without NP }}^{2} \mathrm{~d} V},
$$

where $\varepsilon_{m}^{\prime \prime}$ is the imaginary part of the dielectric function of $\mathrm{Si}$ modified for the case with metallic nanoparticles, whereas $\varepsilon_{0}^{\prime \prime}$ is the imaginary part of the dielectric function of Si without any metallic components (the latter after [8]). The ratio $\mathcal{A}$ given by Eq. (8) displays the role of plasmon mediated energy absorption in the semiconductor substrate. The difference between denominator and numerator in Eq. (8) does not resolve only to different imaginary part of the dielectric constant but also concerns the electric field $E$ with (without) concentration due to the presence of metallic particles. In Fig. 1 we compare the related photocurrent gain due to local increase of $E$ with $\varepsilon_{0}^{\prime \prime}$ and with that $\varepsilon_{m}^{\prime \prime}$ found via the Fermi golden rule. The latter appears to be most important factor to give realistic increase of the photocurrent (in Fig. 1 for nanoparticles with $a=25,40,50 \mathrm{~nm}(\mathrm{Au})$ with planar densities adjusted to the same total mass of the metal). The short circuit current $I_{S C}$ was obtained by integration of absorption enhancement over the AM1.5G solar spectrum assuming 100\% charge separation efficiency.

In order to estimate the difference between two situations: (1) concentration of electric field near metallic nanoparticles only, (2) inclusion of the near field coupling between plasmons and Si substrate, we have performed a numerical modeling of plasmon mediated photovoltaic effect using the quantum modified dielectric function of Si caused by the top-deposited metallic nanoparticles in comparison to the measured in bulk dielectric function of Si without any metallic components, the latter taken from Ref. [8]. The dielectric function of Au was described by the Drude model with parameters $\varepsilon_{\infty}=9, \hbar \omega_{p}=9 \mathrm{eV}$ and damping $\gamma$ defined as in Ref. [9].

In Fig. 1 we compare the gain due to mediation of surface plasmons in metallic nanospheres deposited on the top surface of Si photodiode: left - when only concentration of electric field near the metallic nanoparticles is accounted for (in standard Comsol calculus), right - 

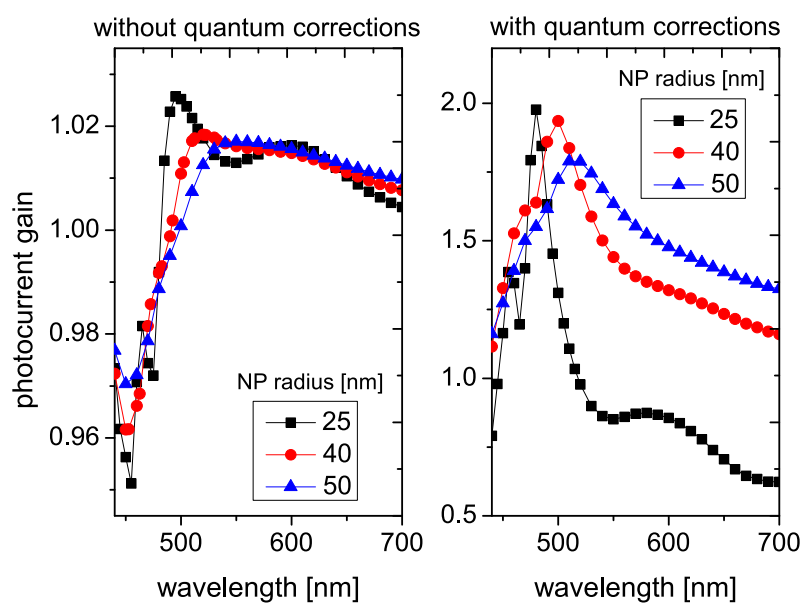

Fig. 1. The comparison of photocurrent gain calculated for nanoparticle radii 25,40 , and $50 \mathrm{~nm}$ and planar concentrations $n_{s}=6.6 \times 10^{8}, n_{s}=1.6 \times 10^{8}$, and $n_{s}=0.8 \times 10^{8} \mathrm{~cm}^{-2}$ with dielectric function of Si taken from experiment [8] (left part) and with the dielectric function of Si modified upon the QM Fermi golden rule scheme (right part).
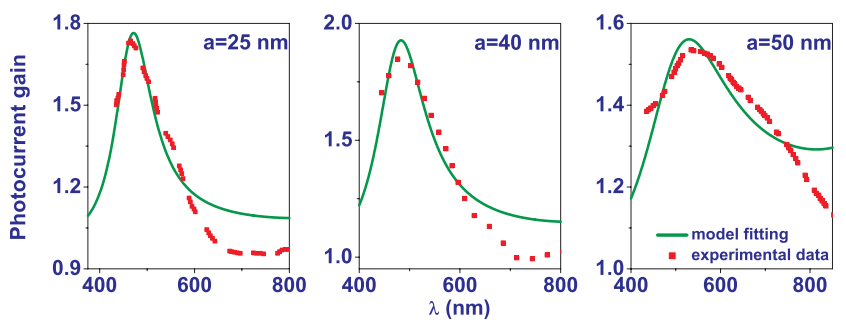

Fig. 2. Dependence of the normalized photocurrent $\frac{I^{\prime}}{I}(\lambda)$ given by the analytical formulae (3) and (4): comparison with the experimental data (red) after [1] for: (left) $a=25 \mathrm{~nm}$ and MNPs concentration $n_{s}=6.6 \times$ $10^{8} \mathrm{~cm}^{-2}$; (center) $a=40 \mathrm{~nm}$ and $n_{s}=1.6 \times 10^{8} \mathrm{~cm}^{-2}$; (right) $a=50 \mathrm{~nm}$ and $n_{s}=0.8 \times 10^{8} \mathrm{~cm}^{-2}(H=3 \mu \mathrm{m})$.

when the enhancement of the imaginary part of the dielectric function of Si modified by metallic nanoparticles is taken into account. The difference is pronounced, as visible in Fig. 1, and it indicates that it is impossible to explain experimental data as shown in Fig. 2 by standard Comsol only. An inclusion of quantum corrections is thus necessary to avoid a large error.

\section{Results}

Comparison of the photocurrent gain (calculated for dielectric function of Si taken from an experiment and the one obtained analytically with quantum corrections) with the results of the experiment [1] (see Fig. 1 and Fig. 3) suggests that e-m field concentration near the MNP results is too small overall photocurrent enhancement apparently insufficient to explain the observed data. For metallic nanoparticles planar density of the order $n_{s}=10^{8} \mathrm{~cm}^{-2}$ [1], the maximum of photocurrent gain calculated with non-modified $\mathrm{Si}$ dielectric function
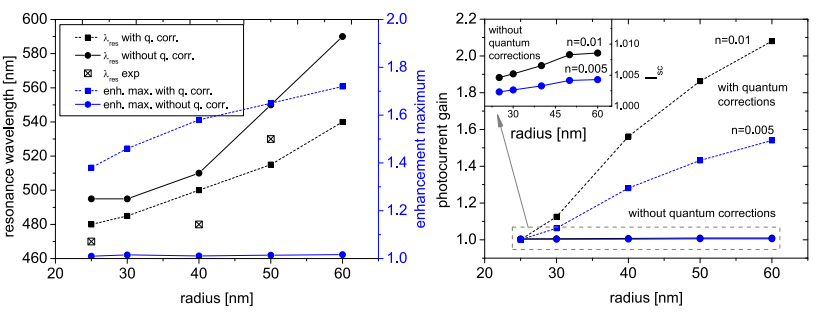

Fig. 3. (left) The resonance wavelength and the maximal photocurrent gain enhancement (at the resonance) as a function of metallic nanoparticle radii for constant relative coverage $n=0.005$ of the semiconductor surface by metallic components (without quantum corrections and modified upon the Fermi golden rule). Experimental data after [1] shown for comparison. (right) Comparison of the total photocurrent gain, integrated over the AM1.5G solar spectrum, calculated for two relative nanoparticle coverages $n=0.01$ and $n=0.005$ for Si dielectric functions as in the left part.

does not exceed the value of $\frac{I^{\prime}}{I}=1.05$. In the case of the modified Si dielectric function, the photocurrent gain is significantly larger and the spectra height, shape, and width are in the satisfactory coincidence with the experimental data. The same refers to our analytical formulae (see Fig. 2). However, we must emphasize the role of the parameter $\beta$ in the theoretical formula (see Eqs. (3) and (4)), which accounts for all details neglected in the model and resulting in reducing of the total photocurrent gain, such as the nanoparticle deposition type or semiconductor surface property modifications induced by metallic components. One can assess $\beta \sim \frac{h}{a}$ (where $h$ is the effective range of the near field coupling) for the metallic nanoparticle deposited atop of the semiconductor, whereas in the case of the nanoparticle entirely embedded in the semiconductor, the parameter $\beta$ would be much larger. After comparison with the experimental data the parameter $\beta$ was obtained to be of order of $\beta=0.002$.

In must be also emphasized that using the nonmodified dielectric function of $\mathrm{Si}$ and thus taking into account solely the enhancement resulting from the locally concentrated e-m field the observed photocurrent gain is hard to be explained and the inclusion of quantum correction is apparently necessary. Simultaneously, as shown in Fig. 3, we have obtained the similar shift of the resonance maximum as shift observed in the experiment towards longer wavelengths with an increase of the metallic nanoparticles radii, as well as an increase of the total photocurrent gain with an enlargement of the semiconductor surface coverage $\left(n=\frac{\pi a^{2}}{A_{0}}\right.$, where $A_{0}$ is the surface of the computational cell for Comsol modeling).

\section{Summary}

We have presented an analytical and numerical approach to modeling of plasmon improved solar cells including quantum corrections arising from coupling between plasmons and semiconductor band electrons captured within the quantum Fermi golden rule approach. This opens 
new area for fine-tuning and optimization of metallic nanomodified solar cell efficiency enhancement based on numerical modeling with inclusion of important quantum mechanical effects, previously completely neglected. This result might be also found as a contribution to further development of the Comsol modeling environment.

\section{Acknowledgments}

A part of this work was supported by the COST Action MP1406 "Multiscale in modelling and validation for solar photovoltaics (MultiscaleSolar)". W.J. acknowledges funding by Polish Ministry of Science (MNiSW grant no. 0731/E-366/STYP/1/11/2016/2017).

\section{References}

[1] D.M. Schaadt, B. Feng, E.T. Yu, Appl. Phys. Lett. 86, 063106 (2005).

[2] M.-J. Jeng, Z.-Y. Chen, Y.-L. Xiao, L.-B. Chang, J. Ao, Y. Sun, E. Popko, W. Jacak, L. Chow, Materials $\mathbf{8}, 6761$ (2015).
[3] N. Spalatu, J. Hiie, N. Maticiuc, M. Krunks, A. Katerski, V. Mikli, I. Sildos, Appl. Surf. Sci. 350, 69 (2015).

[4] J.G. Smith, J.A. Faucheaux, P.K. Jain, Nano Today 10, 67 (2015).

[5] Y.A. Akimov, W.S. Koh, S.Y. Sian, S. Ren, Appl. Phys. Lett. 96, 073111 (2010).

[6] Y.A. Akimov, W.S. Koh, Plasmonics 6, 155 (2011).

[7] S. Wu, W. Wang, K. Reinhardt, Y. Lu, S. Chen, J. Nanophoton. 4, 043515 (2010).

[8] D.E. Aspnes, A.A. Studna, Phys. Rev. B 27, 985 (1983).

[9] W. Jacak, E. Popko, A. Henrykowski, E. Zielony, K. Gwóźdź, R. Pietruszka, G. Luka, B. Witkowski, L. Wachnicki, L.-B. Chang, M.-J. Jeng, Solar En. Mater. Solar Cells 147, 1 (2016).

[10] D. Pines, Elementary Excitations in Solids, ABP Perseus Books, Reading (MA) 1999.

[11] W. Jacak, J. Krasnyj, J. Jacak, R. Gonczarek, A. Chepok, L. Jacak, D.Z. Hu, D. Schaadt, J. Appl. Phys. 107, 124317 (2010).

[12] L.D. Landau, E.M. Lifshitz, Electrodynamics of Continuous Media, in series: Course of Theoretical Physics, Vol. 8, Pergamon Press, New York 1960. 